\title{
Archivos \\ de Cardiología de México

\section{Registro de síndromes coronarios agudos sin elevación del segmento ST en un centro hospitalario de tercer nivel de atención (estudio RESCATA-SEST)}

\author{
José Angel González-Pliego ${ }^{a, *}$, Gonzalo Israel Gutiérrez-Díaz ${ }^{a}$, Alfredo Celis ${ }^{b}$ \\ y Diego Armando Gudiño-Amezcua ${ }^{a}$
}

\begin{abstract}
a Departamento de Cardiología Clínica, Unidad Médica de Alta Especialidad, Hospital de Especialidades, Centro Médico Nacional de Occidente, Instituto Mexicano del Seguro Social, Guadalajara, Jalisco, México

b Unidad de Investigación en Epidemiología Clínica, Unidad Médica de Alta Especialidad, Hospital de Especialidades, Centro Médico Nacional de Occidente, Instituto Mexicano del Seguro Social, Guadalajara, Jalisco, México
\end{abstract}

Recibido el 26 de marzo de 2013; aceptado el 25 de noviembre de 2013

\author{
PALABRAS CLAVE \\ Angina inestable; \\ Infarto de miocardio \\ sin elevación del ST; \\ Síndrome coronario \\ agudo; \\ Síndrome coronario \\ agudo sin elevación \\ del ST; \\ Registros; \\ México
}

\begin{abstract}
Resumen
Objetivo: Describir el perfil clinicoepidemiológico y el proceso de atención del síndrome coronario agudo sin elevación del segmento ST en un hospital de tercer nivel.

Método: Se analiza la información clínica, la estratificación de riesgo, la terapia de revascularización y los hábitos de prescripción al egreso de los casos con síndrome coronario agudo sin elevación del segmento ST atendidos en un año.

Resultados: Se incluyeron 283 pacientes con una edad media de 58 años; el 63\%, masculino. La mayoría (88.6\%) de los casos ocurrió entre los 50 y 59 años. La hipertensión arterial fue el factor de riesgo predominante. El $82.5 \%$ de los sujetos tuvo índice TIMI de riesgo bajo-intermedio. En el $37 \%$ de los pacientes hubo isquemia residual y en $80(70 \%)$ se demostraron obstrucciones coronarias. Setenta y dos pacientes $(90 \%)$ fueron revascularizados con stent, principalmente farmacológico (87.5\%). Más del $90 \%$ de los casos recibió estatina y antiplaquetarios al egreso; otros medicamentos se indicaron en poco más del $50 \%$.

Conclusiones: En la población estudiada, el síndrome coronario agudo sin elevación del ST predomina en hombres relativamente jóvenes e hipertensos. Estratificar el riesgo, buscar isquemia residual y revascularizar con stent farmacológico son prácticas comunes; el cumplimiento de las recomendaciones basadas en la evidencia es subóptimo.

(c) 2013 Instituto Nacional de Cardiología Ignacio Chávez. Publicado por Masson Doyma México S.A. Todos los derechos reservados.
\end{abstract}

\footnotetext{
* Autor para correspondencia: Departamento de Cardiología Clínica, Unidad Médica de Alta Especialidad, Hospital de Especialidades, Centro Médico Nacional de Occidente, Belisario Domínguez n. ${ }^{\circ}$. 1000, Col. Independencia, CP 44349, Guadalajara, Jalisco, México. Teléfono oficina: +0133 361700 60, ext. 31523; Teléfono: +044 3336 624819; fax: +0133 36474260 .
}

Correo electrónico: gopa1@att.net.mx (J.A. González-Pliego). 


\section{KEYWORDS}

Unstable angina; Non-ST elevation acute myocardial infarction; Acute coronary syndromes; Non-ST elevation acute coronary syndromes; Registries; Mexico

\section{Registry of non-ST elevation acute coronary syndromes in a tertiary hospital (RESCATA-SEST registry)}

\begin{abstract}
Objective: To describe the clinical-epidemiologic profile and the process of care of the non-ST elevation acute coronary syndromes in a tertiary hospital.

Method: We analyzed the clinical information, the risk stratification and diagnostic methods, the revascularization therapy and the prescription trends at discharge, of patients with non-ST elevation acute coronary syndromes cared for in one year.

Results: Two hundred and eighty-three patients with mean age of 58 years were included (63\% men). The largest number of non-ST elevation acute coronary syndromes (88.6\%) was found between 50 to 59 years of age. The most common risk factor was hypertension; $82.5 \%$ of the patients had a low-intermediate TIMI score; residual ischemia was demonstrated in $37 \%$ and coronary obstructions were seen in 80 patients (70\%). In $90 \%$, a percutaneous coronary intervention was performed, mainly with drug-eluting Stents (87.5\%). At discharge, even though antiplatelet agents and statins were prescribed in more than $90 \%$, other drugs were indicated in a few more than $50 \%$ of patients.

Conclusions: In this population, non-ST elevation acute coronary syndromes predominates in relatively young men, often with hypertension. To stratify risk, to look for residual ischemia and to revascularize with drug-eluting stents are common practices, but the evidence-based guidelines compliance is still suboptimal.

(C) 2013 Instituto Nacional de Cardiología Ignacio Chávez. Published by Masson Doyma México S.A. All rights reserved.
\end{abstract}

\section{Introducción}

El pronóstico del síndrome coronario agudo sin elevación del segmento ST (SCASEST), conformado por la angina inestable (AI) y el infarto de miocardio sin elevación del segmento ST (IMSEST) depende de su manejo inmediato y de las medidas de prevención secundaria; la falta de adhesión a las recomendaciones específicas, basadas en evidencia científica y ampliamente difundidas ${ }^{1,2}$, es un factor que impide mejorar las expectativas de la población afectada por este síndrome clínico. En nuestro medio supone un gran impacto médico, económico y social y su presencia ha sido claramente documentada ${ }^{3,4}$.

En 2 registros nacionales ${ }^{3,5}$ se han comunicado las características clínicas, el manejo y la evolución hospitalaria de estas formas de presentación de la cardiopatía isquémica entre los años 1998 y $2001^{3}$, y entre diciembre de 2002 y noviembre de $2003^{5}$. Uno más evaluó el riesgo de los síndromes coronarios agudos utilizando la escala del Global Registry of Acute Coronary Events ${ }^{6}$ en hospitales de tercer nivel de atención del Instituto Mexicano del Seguro Social ${ }^{7}$.

El propósito de este trabajo es describir el perfil clinicoepidemiológico y el proceso de atención del SCASEST en un hospital de tercer nivel de atención.

\section{Objetivo}

Describir las características clínicas y epidemiológicas, el uso de métodos de estratificación de riesgo de insuficiencia cardiaca y de mortalidad a corto plazo, la aplicación de biomarcadores, los hallazgos electrocardiográficos, la búsqueda de isquemia residual mediante ergometría o ecocardiografía de estrés, la realización de cateterismo diagnóstico y terapéutico, y los hábitos de prescripción de tratamiento farmacológico al egreso hospitalario, en los pacientes con los diagnósticos de Al o IMSEST, atendidos en el Departamento de Cardiología Clínica, del Hospital de Especialidades del Centro Médico Nacional de Occidente, del Instituto Mexicano del Seguro Social, entre el 1 de marzo de 2010 y el 28 de febrero del 2011.

\section{Métodos}

Para conocer las características epidemiológicas, clínicas, de estudio y tratamiento de los pacientes con SCASEST egresados del Departamento de Cardiología Clínica de nuestro centro hospitalario, entre el 1 de marzo de 2010 y el 28 de febrero de 2011, se efectuó el registro de la información en un formato previamente diseñado. El universo de estudio se conformó con los pacientes que cumplieran los siguientes criterios de inclusión: hombres y mujeres mayores de 18 años cuyo diagnóstico de egreso fuera Al o IMSEST, de acuerdo a los criterios convencionales ${ }^{1,2,8}$; se excluyeron los pacientes cuya fecha de internamiento estuviera fuera del periodo establecido para el presente registro o cuyo diagnóstico no correspondiera a Al o IMSEST, y se eliminaron los pacientes que, teniendo diagnóstico de algún SCASEST, no contaran con los datos mínimos requeridos en el expediente al egreso hospitalario.

Se recabó del expediente clínico la información sobre diagnóstico nosológico, sexo, edad, factores de riesgo cardiovascular, clasificación de Killip-Kimball ${ }^{9}$ en los casos con IMSEST, índice de riesgo Thrombolisys in Myocardial Infarction (TIMI) ()$^{10}$, los resultados de glucosa, creatinina, fracción miocárdica de creatinfosfocinasa (CPK-MB) y troponina I; la información relativa a trastornos del ritmo o 
de la conducción y de necrosis en el electrocardiograma; los resultados de prueba de esfuerzo (PE) en banda sin fin, o de ecocardiograma de estrés para la búsqueda de isquemia residual, métodos disponibles en nuestro hospital para su utilización después de la fase aguda del episodio isquémico, durante la permanencia de los pacientes en el mismo (considerando la primera como positiva al existir depresión $\geq 1 \mathrm{~mm}$ del segmento ST a los $80 \mathrm{~ms}$ en al menos 2 derivaciones contiguas, o por la presencia de angina durante o después del ejercicio, y la segunda al demostrarse anormalidades de la contractilidad tras la infusión de dobutamina intravenosa); la fracción de expulsión del ventrículo izquierdo y la variedad de disfunción diastólica por ecocardiografía; los resultados de la coronariografía diagnóstica, la realización y tipo de revascularización, la utilización de stents intracoronarios y los fármacos prescritos al egreso hospitalario. Todo ello fue volcado en una base de datos electrónica especialmente diseñada para este registro, utilizando el software Microsoft Visual Fox Pro 9.0.

Para una estimación de distribución de variables cualitativas con una precisión de $\pm 6 \%$, con un intervalo de confianza del $95 \%$, se calculó un tamaño de muestra superior a 280 pacientes, conformada con el total de pacientes atendidos durante un año en el área de trabajo mencionada. La edad se expresó como promedio e intervalo y se dividió proporcionalmente por décadas; se calculó la proporción de cada factor de riesgo, del perfil de riesgo TIMI en toda la población y de la gravedad de la insuficiencia cardiaca mediante la escala Killip-Kimball en los pacientes con IMSEST. Los resultados de laboratorio se dividieron en intervalos y se expresaron en proporciones; se determinaron los porcentajes de los hallazgos del electrocardiograma en reposo, el resultado de isquemia en la PE, la calificación en la escala de riesgo de Duke y el número de segmentos isquémicos en el ecocardiograma; la fracción de expulsión se expresó en intervalos y proporciones y los diferentes tipos de disfunción diastólica en porcentajes; para los casos con angiografía coronaria se determinaron proporcionalmente el tipo de indicación, el número de arterias con obstrucción significativa y el número de stents por vaso afectado; se calculó la proporción cuya prescripción al egreso hospitalario incluyera aspirina, clopidogrel, estatina, bloqueadores beta, inhibidores de la enzima convertidora de angiotensina o antagonistas de los receptores de la angiotensina ॥.

El proyecto de investigación fue aprobado por la Comisión de Investigación del Hospital de Especialidades del Centro Médico Nacional de Occidente del Instituto Mexicano del Seguro Social (n. ${ }^{\circ}$ de registro: R-2012-1301-27). Teniendo en cuenta que la investigación se basó exclusivamente en datos registrados en la historia clínica de los pacientes estudiados, y por tratarse de una investigación clasificada como «sin riesgo», los investigadores solicitaron autorización al comité de ética para omitir la solicitud del «consentimiento informado» por parte de los pacientes, en estricta adhesión a los artículos 17 y 23 del Reglamento de la Ley General de Salud en Materia de Investigación para la Salud.

\section{Resultados}

Se incluyeron para el procesamiento y análisis 283 pacientes con diagnóstico de Al e IMSEST. La mayoría fueron hombres
Tabla 1 Datos demográficos y clínicos en síndromes coronarios agudos sin elevación del segmento ST $(\mathrm{N}=283)$

\begin{tabular}{|c|c|c|}
\hline \multirow[t]{2}{*}{$\begin{array}{l}\text { Hombres, } n(\%) \\
\text { Edad (promedio/intervalo) }\end{array}$} & \multicolumn{2}{|c|}{$178(63)$} \\
\hline & $\mathrm{n}$ & $(\%)$ \\
\hline \multicolumn{3}{|l|}{ Edad por décadas } \\
\hline$<30 a$ & 1 & $(0.35)$ \\
\hline $30-39 a$ & 5 & $(1.76)$ \\
\hline $40-49 a$ & 16 & $(5.6)$ \\
\hline $50-59 a$ & 251 & $(88.6)$ \\
\hline $60-69 a$ & 3 & $(1.06)$ \\
\hline $70-79 a$ & 2 & $(0.69)$ \\
\hline$>80 \mathrm{a}$ & 5 & $(1.76)$ \\
\hline \multicolumn{3}{|l|}{ Tipo de SCASEST } \\
\hline Angina inestable & 153 & $(54.0)$ \\
\hline IMSEST & 130 & $(46.0)$ \\
\hline \multicolumn{3}{|c|}{ Factores de riesgo cardiovascular } \\
\hline Hipertensión arterial & 217 & $(76.7)$ \\
\hline Dislipidemia & 171 & $(60.4)$ \\
\hline Sedentarismo & 161 & $(57.2)$ \\
\hline Tabaquismo & 153 & $(54.4)$ \\
\hline Diabetes mellitus & 118 & $(42.0)$ \\
\hline Menopausia & 92 & $(87.0)^{\mathrm{a}}$ \\
\hline Historia familiar & 66 & $(23.3)$ \\
\hline Síndrome metabólico & 25 & $(9.2)$ \\
\hline
\end{tabular}

IMSEST: infarto de miocardio sin elevación del segmento ST; SCASEST: síndrome coronario agudo sin elevación del segmento ST.

a Del total femenino.

y destaca el mayor número de casos ocurridos en la sexta década de la vida, con leve predominio de la Al.

En las mujeres el factor de riesgo más común fue la menopausia, pero se observó una elevada prevalencia de hipertensión arterial, dislipidemia, sedentarismo, tabaquismo y diabetes mellitus (tabla 1). La mayoría de los casos con IMSEST se situó en la clase I de Killip-Kimball y 2 terceras partes de la población con SCASEST tuvo riesgo TIMI $\leq 3$ (tabla 2). La distribución y el porcentaje de casos según los

Tabla 2 Gravedad de insuficiencia cardiaca y riesgo TIMI en síndromes coronarios agudos sin elevación del segmento ST

\begin{tabular}{|c|c|c|}
\hline & $\mathrm{n}$ & $(\%)$ \\
\hline \multicolumn{3}{|c|}{ Clasificación de Killip-Kimball $(N=130)$} \\
\hline I & 92 & $(71.0)$ \\
\hline II & 30 & $(23.0)$ \\
\hline III & 8 & $(6.0)$ \\
\hline \multicolumn{3}{|c|}{ Riesgo TIMI $(\mathrm{N}=283)$} \\
\hline 1 & 22 & $(7.7)$ \\
\hline 2 & 61 & $(21.5)$ \\
\hline 3 & 92 & $(32.5)$ \\
\hline 4 & 59 & $(20.8)$ \\
\hline 5 & 34 & $(12.0)$ \\
\hline 6 & 15 & $(5.3)$ \\
\hline
\end{tabular}

TIMI: Thrombolisys in Myocardial Infarction. 
Tabla 3 Distribución porcentual de biomarcadores de daño miocárdico y química sanguínea en síndromes coronarios agudos sin elevación del segmento ST $(\mathrm{N}=283)$

\begin{tabular}{|c|c|c|c|}
\hline CPK-MB & Intervalos (UI) & $\mathrm{n}$ & $(\%)$ \\
\hline & $16-100$ & 99 & $(35.3)$ \\
\hline & $101-500$ & 25 & $(8.5)$ \\
\hline & $>1,000$ & 4 & (1.4) \\
\hline & Sin incremento & 155 & $(54.8)$ \\
\hline \multirow[t]{7}{*}{ Troponina I } & Intervalos $(\mu \mathrm{g} / \mathrm{L})$ & $\mathrm{n}$ & $(\%)$ \\
\hline & $0.01-0.99$ & 45 & $(15.90)$ \\
\hline & $1.00-4.99$ & 90 & $(31.80)$ \\
\hline & $5.00-9.99$ & 30 & $(10.60)$ \\
\hline & $10.0-14.99$ & 3 & $(1.06)$ \\
\hline & $>30$ & 7 & $(2.47)$ \\
\hline & Sin incremento & 108 & $(38.16)$ \\
\hline \multirow[t]{6}{*}{ Glucosa } & Intervalos (mg/dL) & $\mathrm{n}$ & $(\%)$ \\
\hline & $70-110$ & 122 & $(43.8)$ \\
\hline & $111-126$ & 12 & (3.9) \\
\hline & $127-200$ & 39 & (13.4) \\
\hline & $201-400$ & 15 & $(5.3)$ \\
\hline & Sin datos & 95 & $(33.6)$ \\
\hline \multirow[t]{5}{*}{ Creatinina } & Intervalos (mg/dL) & $\mathrm{n}$ & $(\%)$ \\
\hline & $0.5-1.5$ & 181 & $(64.0)$ \\
\hline & $1.51-2.0$ & 9 & $(3.2)$ \\
\hline & $>2.01$ & 89 & (31.4) \\
\hline & Sin datos & 4 & (1.4) \\
\hline
\end{tabular}

CPK-MB: fracción miocárdica de creatinfosfocinasa.

rangos de los biomarcadores de daño miocárdico, la glucosa y la creatinina revelan que en una importante proporción no se detectó incremento de la CPK-MB y de la I o que este se mantuvo en los valores bajos de su determinación. Destaca la glucosa normal en la mayoría y la creatinina sérica superior a lo normal en un tercio de los pacientes (tabla 3).

Nueve de cada 10 sujetos con SCASEST tenían ritmo sinusal, y las arritmias y anomalías de la conducción eléctrica documentadas se distribuyeron así: extrasístoles ventriculares, 17 casos (6.0\%); fibrilación auricular, 7 casos $(2.4 \%)$; taquicardia y fibrilación ventricular, un caso cada una (0.3\%); bloqueo de rama izquierda, 22 casos (7.8\%); bloqueo de rama derecha, 27 casos (9.5\%) y bloqueo auriculoventricular completo, 4 casos (1.4\%). Una imagen de necrosis antigua en la cara anterior se observó en 31 pacientes $(10.9 \%)$ y en la inferior en $40(14.1 \%)$. En 3 sujetos $(1.06 \%)$ se apreció una zona de necrosis actual.

Después del episodio agudo del SCASEST, la búsqueda de isquemia residual se efectuó mediante PE en banda sin fin en 165 casos, utilizando los protocolos de Bruce, Bruce modificado o Naugthon, y a través de ecocardiografía en 79 pacientes. Casi la tercera parte de las pruebas ergométricas reveló isquemia miocárdica y en la escala de Duke solo una pequeña proporción se situó en riesgo elevado; los segmentos isquémicos detectados mediante el método de imagen fueron menos de 3 en la mayoría de los sujetos estudiados con este método. La caracterización ecocardiográfica de la
Tabla 4 Función ventricular izquierda por ecocardiografía en casos con síndromes coronarios agudos sin elevación del segmento ST $(\mathrm{N}=153$

\begin{tabular}{lrr}
\hline & $\mathrm{n}$ & $\%$ \\
\hline Función sistólica & & \\
$\quad \begin{array}{l}\text { Fracción de expulsión (\%) } \\
\quad 255\end{array}$ & 96 & $(62.7)$ \\
$\quad 45-55$ & 37 & $(24.1)$ \\
$30-44$ & 14 & $(9.1)$ \\
$<30$ & 6 & $(3.9)$ \\
Función diastólica & & \\
Normal & 76 & $(49.36)$ \\
Disfunción tipo I & 54 & $(35.44)$ \\
Disfunción tipo II & 18 & $(12.02)$ \\
Disfunción tipo III & 5 & $(3.16)$ \\
\hline
\end{tabular}

función ventricular izquierda muestra que predominaron la alteración de la relajación y la fracción de expulsión normal (tabla 4).

Se efectuó angiografía coronaria en 114 (40.2\%) pacientes durante la hospitalización; en 10 de ellos (8.7\%) dentro de las primeras $24 \mathrm{~h}$, pero en la mayoría después de haberse demostrado isquemia residual en PE (48 casos; $42.1 \%$ ) o en ecocardiograma con infusión de dobutamina (56 casos; $49.1 \%)$.

Considerando significativas las obstrucciones coronarias $\geq 70 \%$, el número de casos por vasos afectados se distribuyó de la siguiente manera: un vaso, 41 casos (35.9\%); 2 vasos, 27 casos (23.6\%); 3 vasos, 12 casos (10.5\%). En el resto de los pacientes sometidos al estudio invasivo (34; 29.8\%), no se encontraron lesiones significativas (tabla 5).

La distribución específica de los casos con obstrucción univascular significativa y la de sus combinaciones cuando hubo afectación de 2 o de tres 3 arterias coronarias señala que la enfermedad más común en forma aislada o en combinación fue la de la descendente anterior, que además recibió la mayor parte de los stents intracoronarios colocados (tabla 6). El tipo más comúnmente utilizado fue el

Tabla 5 Distribución de casos según el número de vasos y la arteria afectada en síndromes coronarios agudos sin elevación del segmento ST $(\mathrm{N}=114)$

\begin{tabular}{|c|c|c|}
\hline Número de vasos & $\mathrm{n}$ & $(\%)$ \\
\hline 0 & 34 & $(100)$ \\
\hline \multicolumn{3}{|l|}{1} \\
\hline DA & 21 & $(51.21)$ \\
\hline$C x$ & 13 & $(31.70)$ \\
\hline$C D$ & 7 & $(17.07)$ \\
\hline \multicolumn{3}{|l|}{2} \\
\hline $\mathrm{DA}+\mathrm{CD}$ & 4 & $(14.81)$ \\
\hline $\mathrm{DA}+\mathrm{Cx}$ & 19 & $(70.37)$ \\
\hline$C x+C D$ & 4 & $(14.81)$ \\
\hline \multicolumn{3}{|l|}{3} \\
\hline$D A+C x+C D$ & 12 & $(100)$ \\
\hline
\end{tabular}

CD: coronaria derecha; Cx: circunfleja; DA: descendente anterior. 
Tabla 6 Intervencionismo coronario percutáneo con endoprótesis en síndromes coronarios agudos sin elevación del segmento ST $(\mathrm{N}=72)$

\begin{tabular}{lrr}
\hline Arteria afectada & \multicolumn{2}{c}{ Pacientes en los que } \\
se colocó stent, $\mathrm{n}$ & $(\%)$ \\
\hline DA & 26 & $(36.11)$ \\
CX & 17 & $(23.61)$ \\
CD & 9 & $(12.5)$ \\
DA + CD & 12 & $(16.66)$ \\
DA + Cx & 5 & $(6.94)$ \\
CX +CD & 3 & $(4.16)$ \\
\hline
\end{tabular}

CD: coronaria derecha; $\mathrm{Cx}$ : circunfleja; DA: descendente anterior.

recubierto con fármaco $(n=63 ; 87.5 \%)$, en tanto que el metálico se usó en 9 casos (12.5\%). Al egreso hospitalario los fármacos más prescritos fueron aspirina y estatinas $(n=263$; 92.9\% y $n=265 ; 93.6 \%$ respectivamente), pero resalta que el clopidogrel se indicó en casi el total de los casos en los que se implantó un stent intracoronario $(n=71 ; 98.6 \%)$. Entre la población total, los bloqueadores beta, el clopidogrel y un inhibidor de la enzima convertidora de la angiotensina o un antagonista de receptores de la angiotensina II se indicaron en el $66,58.6$ y $54 \%$ de los enfermos respectivamente.

\section{Discusión}

En esta población, los SCASEST ocurren preferentemente en hombres en la sexta década de la vida, más probablemente hipertensos, dislipidémicos, sedentarios, fumadores, diabéticos y con evidencia de disfunción renal. En la mayoría se investiga isquemia residual y se efectúa revascularización percutánea, preferentemente con stents recubiertos.

\section{Datos demográficos}

La mayor proporción de casos con SCASEST en el presente registro ocurrió en hombres en la sexta década de la vida. Este hallazgo es similar al informado en el registro RENASICA ${ }^{3}$. En contraste, la edad promedio de presentación del SCASEST en este registro fue 6 años menor que en otros 2 estudios mexicanos ${ }^{5,7}$, lo que podría indicar que la isquemia miocárdica ocurre en forma aguda cada vez a edades más tempranas. Este hallazgo parece indicar el avance imparable de esta variedad de presentación de miocardio isquémico en hombres mexicanos más jóvenes, incluso cuando se comparan con sujetos hispanos y anglosajones con la misma afección ${ }^{11}$.

La hipertensión arterial se registró en el $75 \%$ de los sujetos, cifra muy similar a la encontrada en los casos con SCASEST del Renasca-IMSS ${ }^{7}$ y que contrasta con el tabaquismo como el factor más prevalente en el RENASICA ${ }^{3}$, y el más común en ese registro respecto a casos de otras etnias con esta enfermedad vascular ${ }^{11}$; en nuestra población, como en el Renasca-IMSS ${ }^{7}$, la diabetes mellitus ocupa el cuarto lugar de prevalencia, detrás de la hipertensión, el tabaquismo y la dislipidemia. Otras variables de interés son la menopausia concordante con la edad promedio de la muestra, y la inactividad física en más de la mitad del grupo; ello recuerda el papel del sedentarismo como promotor de riesgo de enfermedad cardiovascular y el efecto protector que proporciona el ejercicio regular, hecho documentado recientemente en población latinoamericana ${ }^{12,13}$.

\section{Estimación de riesgo}

Como en el RENASICA ${ }^{3}$, la mayoría de nuestros casos se situó en estadio Killip-Kimball I (86.8 y 71\% respectivamente); en el RENASICA II se observó una asociación directamente proporcional entre este índice y la mortalidad hospitalaria en aquellos sujetos con infarto de miocardio con elevación del segmento ST (IMCEST), pero no en los de SCASEST ${ }^{5}$; asimismo, en el Renasca-IMSS se notó una ocurrencia mayor de todos los estadios del mismo en los pacientes con IMCEST, respecto a los sujetos con SCASEST ${ }^{7}$.

Utilizando la escala TIMI para Al/IMSEST ${ }^{10}$, el modelo estratificador temprano más sencillo y que permite planear una estrategia terapéutica ${ }^{2}$, casi 2 terceras partes tuvieron un índice de bajo riesgo (menor de 4 puntos), y en el $38.1 \%$ la posibilidad de muerte, infarto miocárdico o revascularización urgente a 14 días fue $\geq 19.9 \%$.

El único registro mexicano que por la naturaleza de su diseño incluyó una escala de calificación de riesgo para todo el espectro de síndromes coronarios agudos fue el RenascaIMSS $^{7}$. En él, la escala del Global Registry of Acute Coronary Events identificó a la mayoría de los casos con > 150 puntos, aunque sin diferencia significativa entre IMCEST y SCASEST; dicho nivel marcó como complicaciones de cuantía en los casos con SCASEST la angina/isquemia recurrente, la falla del ventrículo izquierdo, el choque, las arritmias y el desenlace combinado de estos eventos. Independientemente de la calificación del riesgo, la adhesión a las guías de manejo actuales para este padecimiento mejora significativamente el pronóstico ${ }^{14}$.

\section{Resultados de laboratorio}

La determinación de CPK-MB y de troponina I se efectuó en todos los casos del presente registro, mientras que en el RENASICA se midieron en el $74 \%$ y en el $11.6 \%$ respectivamente $y$ se informaron conjuntamente para todo el espectro de síndromes coronarios agudos ${ }^{3}$; en el análisis comparativo de los casos con SCASEST del RENASICA con hispánicos y sajones de un registro mayor con el mismo padecimiento, la estratificación con troponinas se efectuó solo en la quinta parte de los $\operatorname{casos}^{11}$. En otra observación, la CPK-MB, que se obtuvo en el $90 \%$ de SCASEST, tuvo un incremento $<1.5 x$ en el $70 \%$ de su población y la troponina । solo se determinó en el $20 \%$ de los pacientes ${ }^{5}$.

En este registro, la troponina I, el biomarcador de elección para el diagnóstico de infarto de miocardio cuando su valor rebasa el percentil $99^{15}$, no sufrió incremento o este fue menor de dicho nivel en el $54 \%$ de la población, lo que supone que el resto tuvo necrosis miocárdica sin traducción electrocardiográfica; ello se correlaciona con el comportamiento de la CPK-MB y con el diagnóstico de Al en quienes estos marcadores no se incrementaron.

En un tercio de nuestra casuística se observaron valores de creatinina sugestivos de una tasa de filtración glomerular subnormal, lo que confirma su presencia frecuente en 
esta condición ${ }^{2}$ y que puede significar daño a órgano blanco hipertensivo y/o diabético, o agudización debida a la agresión isquémica del miocardio.

Los valores de glucosa sérica $>110 \mathrm{mg} / \mathrm{dL}$ documentados en la quinta parte de nuestra población recuerdan que la diabetes mellitus se asocia a una evolución desfavorable de la isquemia aguda ${ }^{16}$; las recomendaciones internacionales actuales incluyen el control estricto de la glucemia ${ }^{2}$ y la corrección de la hiperglucemia $>180 \mathrm{mg} / \mathrm{dL}$ evitando la hipoglucemia ${ }^{1}$. La diabetes mellitus se ha incluido, además, entre las variables para las cuales se prefiere una estrategia de manejo invasiva ${ }^{1,2}$.

\section{Datos electrocardiográficos}

Los bloqueos de rama izquierda y derecha ocurrieron en el $7.8 \%$ y el $9.5 \%$ de nuestra casuística, cifras muy similares a las observadas en el mismo grupo de pacientes del registro RENASICA $\|^{5}$. Este dato es relevante porque, cuando estos bloqueos son nuevos o presumiblemente nuevos, conllevan un mayor riesgo a corto plazo de muerte o infarto miocárdico no fatal ${ }^{17,18}$. Si bien no hubo diferencia en la presencia de arritmias ventriculares y bloqueo auriculoventricular completo, la fibrilación auricular se documentó en más del doble de los casos que en el estudio mencionado ${ }^{5}$. Este dato podría asociarse a disfunción diastólica, a su vez relacionada con la alta prevalencia de hipertensión arterial en nuestra población.

Dado que los pacientes con SCASEST muestran en su mayoría cambios inespecíficos del segmento $\mathrm{ST}^{5}$, el análisis electrocardiográfico en nuestro registro se centró en la presencia o no de necrosis antigua. En la cuarta parte de los casos se apreció este hallazgo, sobre todo en la región inferior $y$, aunque lo ordinario es que los sujetos con IMSEST no muestren onda $\mathrm{Q}$, en una minoría se observó este dato «de novo», lo que indica que el diagnóstico inicial de SCASEST puede virar al de IMCEST ${ }^{19}$. Aunque del grupo total del RENASICA II el 43\% tuvo onda Q, no hay referencia a la presencia de necrosis antigua en los pacientes con SCASEST y su descripción se circunscribe a los cambios inespecíficos del ST y onda $\mathrm{T}$ negativa en un $56 \%$ del grupo de alto riesgo con este diagnóstico y a la depresión extensa o no extensa de dicho segmento en una cuarta parte de ellos respectivamente ${ }^{5}$.

\section{Estratificación de riesgo después de la fase aguda}

Elemento crucial para estratificar el riesgo después de la fase aguda del SCASEST, la búsqueda de isquemia con PE $\mathrm{y} / \mathrm{o}$ ecocardiograma de estrés fue superior a la informada previamente en nuestro medio $^{5}$; la primera permitió identificar al $29 \%(n=48)$ de la población con este hallazgo y saber que, con un riesgo intermedio en la escala de Duke, la mayoría $(n=133 ; 80.6 \%)$ estaba en riesgo de mortalidad anual del 1 a $3 \%^{1}$; el ecocardiograma con infusión de dobutamina ${ }^{20}$, método usado rutinariamente en nuestro centro hospitalario para investigar alteraciones de la perfusión miocárdica, reveló isquemia de $\geq 3$ segmentos en 4 de cada 5 casos en los que se empleó $(n=63 ; 79.6 \%)$, lo que se traduce en un riesgo de mortalidad $>3 \%{ }^{1}$. Puesto que no hay una sugerencia formal sobre la elección del estudio no invasivo para la búsqueda de hipoperfusión miocárdica, esta se basa en las características de los pacientes, su respuesta al tratamiento, la experiencia y la disponibilidad del recurso ${ }^{21}$.

Para guiar el tratamiento e influir en la evolución debe conocerse la función ventricular izquierda ${ }^{1,2}$. Para ello, en poco más de la mitad los pacientes de este registro se efectuó un ecocardiograma transtorácico, proporción muy similar a la de otra serie de casos con SCASEST en nuestro entorno ${ }^{5}$. Así, mientras que en la mayoría de los sujetos se documentó una fracción de expulsión normal, un tercio mostró disfunción sistólica moderada que corresponde a riesgo intermedio de mortalidad, y solo en una pequeña proporción se observó un grave abatimiento funcional del ventrículo izquierdo, cuyo riesgo anual de morir es $\geq 3 \%{ }^{1}$.

La disfunción diastólica tipo III o restrictiva, que se asocia a falla cardiaca, mayor remodelado y mayor mortalidad en el infarto de miocardio ${ }^{22}$, se demostró solo excepcionalmente y, con mucho, la variedad más frecuente fue la de tipo ı.

\section{Enfermedad coronaria obstructiva e intervención coronaria percutánea}

El cateterismo cardiaco se efectuó en una proporción menor en nuestra población en comparación con otros registros nacionales $^{3,5}$, lo que refleja una mayor tendencia al manejo conservador inicial y estratificación de riesgo posterior durante la hospitalización. En la mayoría el procedimiento obedeció a la demostración previa de isquemia miocárdica y en el resto se optó por la estrategia invasiva temprana, obedeciendo las recomendaciones actuales ${ }^{1,2}$.

Como ha sido previamente informado ${ }^{2}$, en la tercera parte de nuestros pacientes se demostró afectación univascular; se encontró enfermedad multivascular en otro tercio de los casos y en el resto el árbol coronario fue angiográficamente normal. Destaca la mayor afectación de la arteria descendente anterior en aquellos con enfermedad de uno o 2 vasos.

Nueve de cada 10 sujetos de nuestra población con obstrucciones coronarias fueron tratados con intervención percutánea $y$, de acuerdo a lo sugerido para esta forma de manejo ${ }^{23}$, en todos se colocó por lo menos una endoprótesis; el tipo de stent más usado y que ha demostrado una reducción en la mortalidad a 2 años y menor necesidad de revascularización en casos con IMSEST ${ }^{24,25}$ fue el liberador de fármaco.

Se aplicó solo un stent en la mayoría de los casos revascularizados por vía percutánea y en una cuarta parte se colocaron 2; aunque hubo menos pacientes con enfermedad de un vaso que sujetos en los que se instaló solo una férula intracoronaria, ello obedeció a que se optó por tratar únicamente la lesión culpable en algunos sujetos, postura valida según las recomendaciones vigentes ${ }^{2}$. Como se ha señalado en nuestro medio ${ }^{3}$, la arteria descendente anterior, la más comúnmente lesionada en enfermedad uni o bivascular, fue la que con más frecuencia se revascularizó durante el cateterismo.

\section{Tratamiento farmacológico al egreso hospitalario}

En contraste con la población total de casos con episodios isquémicos agudos informada hace una década ${ }^{3}$, hay dife- 
rencia en los hábitos de prescripción al egreso hospitalario en el presente registro. El uso de aspirina y de un inhibidor de receptor $\mathrm{P}_{2} \mathrm{Y}_{12}$ se incrementó el 20 y $40 \%$ respectivamente, aunque cabe señalar que prácticamente a todos los casos tratados con stent se les indicó clopidogrel al dejar el hospital. A su vez, los bloqueadores beta y los inhibidores de la enzima convertidora de la angiotensina vieron aumentada su prescripción en un 25 y $8 \%$ respectivamente, y las estatinas, que no se prescribían en aquel entonces, se indicaron en casi todos nuestros casos al regresar a su domicilio.

La mejoría en la evolución de los casos con enfermedad isquémica del corazón tratados en el Instituto Mexicano del Seguro Social en los últimos años ${ }^{26}$ se ha explicado por una mejor prevención secundaria, pero, como en otras latitudes ${ }^{27,28}$, el cumplimiento de las metas actuales para todos los pacientes post-SCASEST, es aún incompleto.

\section{Limitaciones}

El sesgo de selección de los pacientes es común en los estudios observacionales tipo registro; el presente es un trabajo de centro hospitalario único y de tercer nivel de atención, por lo que sus resultados solo pueden compararse o contrastarse con otros sitios similares.

\section{Conclusiones}

Los SCASEST predominan en hombres relativamente jóvenes y frecuentemente hipertensos. El riesgo de desenlaces cardiovasculares a corto plazo es generalmente bajo y es muy factible la revascularización percutánea con stents coronarios recubiertos. Para mejorar el pronóstico, debe buscarse la mayor adhesión al tratamiento farmacológico basado en la evidencia y vigente desde hace varios años ${ }^{29}$.

\section{Financiación}

No se recibió patrocinio de ningún tipo para llevar a cabo este estudio/artículo.

\section{Conflicto de intereses}

Los autores declaran no tener ningún conflicto de intereses.

\section{Bibliografía}

1. Jneid H, Anderson JL, Scott WR, et al., American College of Cardiology Foundation; American Heart Association Task Force on Practice Guidelines. 2012 ACCF/AHA focused update of the guideline for the management of patients with unstable angina/non-ST-elevation myocardial infarction (updating the 2007 guideline and replacing the 2011 focused update): a report of the American College of Cardiology Foundation/American Heart Association Task Force on practice guidelines. Circulation. 2012;126:875-910.

2. Hamm CW, Bassand JP, Agewall S, et al., ESC Committee for Practice Guidelines. ESC guidelines for the management of acute coronary syndromes in patients presenting without persistent ST-segment elevation. The Task Force for the management of acute coronary syndromes (ACS) in patients presenting without persistent ST-segment elevation of the European Society of Cardiology (ESC). Eur Heart J. 2011;32:2999-3054.

3. Lupi HE, Por el Grupo Cooperativo RENASICA. El Registro Nacional de los Síndromes Coronarios Agudos (RENASICA). Sociedad Mexicana de Cardiología. Arch Cardiol Mex. 2002;72 Supl 2:S45-64.

4. Principales causas de mortalidad general, 2008 [consultado 5 Dic 2012). Disponible en www.sinais.gob.mx

5. García-Castillo A, Jerjes-Sánchez C, Martínez BP, et al. RENASICA II. Registro Mexicano de Síndromes Coronarios Agudos. Arch Cardiol Mex. 2005;75 Supl. 1:S6-19.

6. Granger CB, Goldberg RJ, Dabbous O, et al. Predictors of hospital mortality in the Global Registry of Acute Coronary Events. Arch Intern Med. 2003;163:2345-53.

7. Borrayo-Sánchez G, Madrid-Miller A, Arriaga-Nava A, et al. Riesgo estratificado de los síndromes coronarios agudos. Resultados del primer Renasca-IMSS. Rev Med Inst Mex Seguro Soc. 2010;48:259-64.

8. Mendis $\mathrm{S}$, Thygesen $\mathrm{K}$, Kuulasmaa $\mathrm{K}$, et al., Writing group on behalf of the participating experts of the WHO consultation for revision of WHO definition of myocardial infarction. World Health Organization definition of myocardial infarction: 2008-09 revision. Int J Epidemiol. 2011;40:139-46.

9. Killip T 3rd, Kimball JT. Treatment of myocardial infarction in a coronary care unit. A two year experience with 250 patients. Am J Cardiol. 1967;20:457-64.

10. Antman EM, Cohen M, Bernink PJ, et al. The TIMI risk score for unstable angina/non-ST elevation MI. JAMA. 2000;284:835-42.

11. Jerjes Sánchez-Diaz C, Garcia-Badillo E, Jerjes SánchezRamírez C, et al. Clinical characteristics, process of care and outcomes among Mexican, Hispanic and non-Hispanic white patients presenting with non-ST elevation acute coronary syndromes: Data from RENASICA and CRUSADE registries. Arch Cardiol Mex. 2012;82:14-21.

12. Lanas F, Avezum A, Bautista LE, et al. Risk factors for acute myocardial infarction in Latin America. The INTERHEART Latin American study. Circulation. 2007;115:1067-74.

13. Smith SC. Risk factors for myocardial infarction in Latin America. Overweight and obesity. Circulation. 2007;115: 1061-3.

14. Gulati M, Patel S, Jaffe AS, et al. Impact of contemporary guideline compliance on risk stratification models for acute coronary syndromes in the Registry of Acute Coronary Syndromes. Am J Cardiol. 2004;94:873-8.

15. Thygesen K, Alpert JS, Jaffe AS, et al. White HD the writing group on behalf of the joint ESC/ACCF/AHA/WHF task force for the universal definition of myocardial infarction. Thirth universal definition of myocardial infarction. Circulation. 2012;126:2020-35.

16. McGuire DK, Emanuelsson H, Granger CB, et al. Influence of diabetes mellitus on clinical outcomes across the spectrum of acute coronary syndromes Findings from the GUSTO-IIb study. GUSTO IIb investigators. Eur Heart J. 2000;21:1750-8.

17. Braunwald E, Mark DB, Jones RH, et al. Unstable angina: Diagnosis and management. En: Clinical practice guideline number 10 AHCPR Publication no 94-0602 Rockville, MD: Agency for Health Care Policy and Research and the National Hearth, Lung, and Blood Institute, Public Health Service, U S Department of Health and Human Services. 1994.

18. Anderson JL, Adams CD, Antman EM, et al. ACC/AHA 2007 guidelines for the management of patients with unstable angina/non-ST-elevation myocardial infarction: a report of the American College of Cardiology/American Heart Association Task Force on Practice Guidelines. Circulation. 2007;116:e148-304.

19. Fox KA, Cokkinos DV, Deckers J, et al. The ENACT study: A pan-European survey of acute coronary syndromes. 
European Network for Acute Coronary Treatment. Eur Heart J. 2000;21:1440-9.

20. Pellikka P, Nagueh SF, Elhendy AA, et al. American Society of Echocardiography recommendations for performance, interpretation, and application of stress echocardiography. J Am Soc Echocardiogr. 2007;20:1321-470.

21. Breall JA, Simons M. Risk stratification after unstable angina or non-ST elevation myocardial infarction. Up to date [consultado 19 Sep 2013]. Disponible en: http://www.uptodate.com/ contents/rik-stratification-after-unstable-angina-or-non-stelevation-myocardial-infarction?source=preview

22. Nagueh SF, Appleton CP, Gillebert TC, et al. Recomendations for the evaluation of left ventricular diastolic function by echocardiography. Eur J Echocardiogr. 2009;22:107-33.

23. Silber S, Albertsson P, Avilés FF, et al. Guidelines for percutaneous coronary intervention. The task force for percutaneous coronary interventions of the European Society of Cardiology. Eur Heart J. 2005;26:804-47.

24. Mauri L, Silbaugh TS, Garg P, et al. Drug-eluting or baremetal stents for acute myocardial infarction. N Engl J Med. 2008;359:1330-42.
25. Levine GN, Bates ER, Blankenship JC, et al. 2011 ACCF/AHA/SCAl Guideline for percutaneous coronary intervention: A report of the American College of Cardiology Foundation/American Heart Association task force on practice guidelines and the Society for Cardiovascular Angiography and Interventions. Circulation. 2011;124:e574-651.

26. Escobedo-de la Peña J, Rodríguez-Abrego G, Buitrón-Granados LV. Morbilidad y mortalidad por cardiopatía isquémica en el Instituto Mexicano del Seguro Social. Estudio ecológico de tendencias de población amparada por el Instituto Mexicano del Seguro Social entre 1990 y 2008. Arch Cardiol Mex. 2010;80:242-8.

27. Bertomeu V, Cordero A, Quiles J, et al. Control de factores de riesgo y tratamiento de los pacientes con cardiopatía isquémica: registro TRECE. Rev Esp Cardiol. 2009;63:807-11.

28. Bhatt DI, Steg PG, Ohman EM, et al. International prevalence, recognition, and treatment of cardiovascular risk factors in outpatients with atherothrombosis. JAMA. 2006;295:180-9.

29. Bassand JP, Hamm CW, Ardissino D, et al. Guidelines for the diagnosis and treatment of non-ST segment elevation acute coronary syndromes. Eur Heart J. 2007;28:1598-660. 